\title{
Minimally invasive treatment of gynecomastia by ultrasound-guided vacuum-assisted excision: report of a case series
}

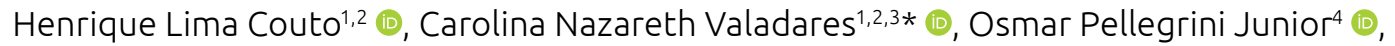

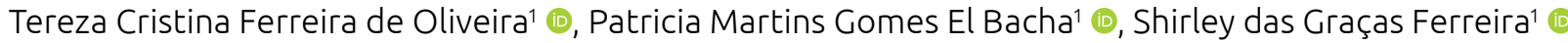

\section{ABSTRACT}

Introduction: Gynecomastia (GM) is a benign proliferation of glandular breast tissue in men. Some cases need surgical intervention. Traditional open surgery by semicircular inferior periareolar incision is the most common surgical approach. In order to obtain better esthetic results, some alternatives to open surgery have been proposed, such as liposuction, endoscopic mastectomy, and vacuum-assisted excision (VAE). Objective: To describe the technical surgical approach of ultrasound-guided VAE of GM and its results from a case series. Method: This is an evaluation of seven GM cases submitted to ultrasound-guided VAE with a 10G needle using the ENCOR ${ }^{\oplus}$ BD whole circumference automated breast biopsy system in Redimasto - Redimama, a Brazilian breast center. The result was considered good or satisfactory when it showed minimal remaining gland, good symmetry, no retraction, necrosis, hypertrophic scar, or displacement of the nipple-areola complex. All patients answered a questionnaire to evaluate their satisfaction and perception of the procedure. Results: Seven (7) patients with Simon grade 1 and 2 bilateral GM underwent ultrasound-guided VAE. No case of displacement, necrosis, or retraction of the nipple-areola complex, post-procedure bleeding, infection, skin necrosis, or asymmetry was detected. No patient reported decrease or change in nipple sensation or erection. All patients had bruises and hematomas that spontaneously resolved within 30 days. All results were considered good or excellent by patients and surgeons. Conclusion: Minimally invasive ultrasound-guided VAE is an excellent alternative for the treatment of GM. It is better indicated for Simon grade 1 and 2 GM, with good and excellent esthetic results, small scar, and low rates of nipple and areolar complications. It allows an outpatient procedure with low morbidity (local anesthesia) and fast recovery.

KEYWORDS: gynecomastia; mammary ultrasonography; interventional ultrasound; needle bipsy.

\section{INTRODUCTION}

Gynecomastia (GM) is a benign proliferation of glandular breast tissue in men $^{1}$. It is the most common male breast disorder, accounting for nearly $60 \%$ of them. It can be unilateral or, most often, bilateral. GM is a common condition with a prevalence of $32 \%$ to $65 \%$, depending on age, and can affect up to $70 \%$ of all pubescent boys ${ }^{2}$. A man's lifespan has three peaks: the first occurs during infancy, the second during puberty, and the third in middle-aged and older men ${ }^{1,2}$. GM in infancy and puberty resolves spontaneously in most cases. Proper investigation is highly recommended among adults and older adults to exclude underlying diseases ${ }^{1}$.
GM typically results from an absolute or relative deficiency of androgen action or excessive estrogen action in the breast tissue $^{2}$. No treatment is necessary for asymptomatic adolescents or men, but it is required when GM is progressive, painful, or causes cosmetic discomfort. It usually resolves by itself or by removing the underlying cause, such as medication, anabolic-androgenic steroid abuse, or treatment of systemic diseases ${ }^{3}$. Medical therapy can also be prescribed for patients with a recent diagnosis within two years -, but is less effective for long-standing GM. Some cases need surgical intervention. According to Simon, GM can be classified into grades ${ }^{4}$ (Table 1).

Traditional open surgery by semicircular inferior periareolar incision is the most common surgical approach, but it may cause

${ }^{1}$ Redimasto, Redimama - Belo Horizonte (MG), Brazil.

${ }^{2}$ Universidade Federal de Minas Gerais - Belo Horizonte (MG), Brazil.

${ }^{3}$ Santa Casa de Belo Horizonte - Belo Horizonte (MG), Brazil.

${ }^{4}$ Hospital da Força Aérea - Brasília (DF), Brazil.

*Corresponding author: carolinanvaladares@gmail.com

Conflict of interests: nothing to declare.

Received on: 11/03/2020. Accepted on: 11/18/2020 
significant morbidities, such as asymmetry, poor scarring, and nipple-areola complex retraction or necrosis ${ }^{5-7}$. In order to obtain better esthetic results, some alternatives to open surgery have been proposed, such as liposuction, endoscopic mastectomy, and vacuum-assisted excision (VAE) ${ }^{7-9}$.

In the last few years, the use of vacuum-assisted devices, originally created to diagnose breast lesions by radiologicallyguided procedures, has shown to be promising in the surgical management of $\mathrm{GM}^{8-12}$.

\section{OBJECTIVE}

To describe the technical surgical approach of ultrasound-guided VAE of GM and its results from a case series.

\section{METHOD}

The study consists of seven GM cases evaluated from December 1, 2018, to December 1, 2019. The patients underwent ultrasoundguided VAE with a $10 \mathrm{G}$ needle using the $\mathrm{ENCOR}^{\oplus} \mathrm{BD}$ whole circumference automated breast biopsy system in Redimasto Redimama, a Brazilian breast center. Before the procedure, all patients were submitted to a clinical evaluation with full history and physical examination by a breast surgeon, as well as mammography, breast ultrasound, and blood tests. All patients signed an informed consent form for the VAE procedure. All procedures were performed by breast surgeons experts in ultrasound-guided VAE. The procedures took place in the breast center, in an outpatient approach, through a $3 \mathrm{~mm}$ incision in each breast, with local anesthesia, using $2 \%$ lidocaine and bupivacaine when necessary, according to the maximum dose

Table 1. Simon grade of gynecomastia.

\begin{tabular}{l|c}
\hline Grade 1 & small breast without excess skin \\
\hline Grade 2 & moderate breast without excess skin \\
\hline Grade 3 & moderate breast with excess skin \\
\hline Grade 4 & large breast with excess skin \\
\hline
\end{tabular}

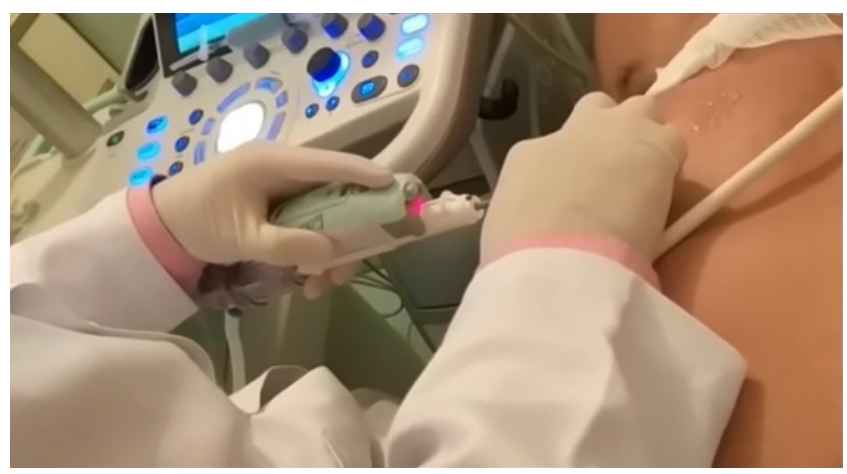

Figure 1. Ultrasound-guided vacuum-assisted excision of gynecomastia: surgical approach. for the patient's weight. No sedation was necessary. After the $10 \mathrm{G}$ needle was introduced and positioned via ultrasound, the automated vacuum device was activated (Figures 1 and 2). The number of fragments extracted from each breast varied according to the surgeon's judgment of each case, taking into account the amount of breast tissue during clinical examination, mammography, and breast ultrasound before surgery, as well as the real-time breast ultrasound evaluation during the procedure. The vacuum method for dense breasts with fine precision was used for all cases. The resection performed left a 1-cm thick gland behind the nipple, just like the standard surgical procedure. At the end of the VAE of the GM, vacuum and manual suction of the residual cavity were performed to avoid or reduce the incidence of postoperative hematomas and bruises. Only one patient had the surgical cavity marked with a metal clip. Mammographic images were obtained one and six months after VAE to evaluate the removal of the glandular tissue (Figure 3). Patients wore a thoracic compression belt for at least 30 days. Follow-up was scheduled at 7 days, 14 days, 1 month, 2 months, and 6 months after the procedure, and consisted of clinical examination, pictures, and survey of the patient's and breast surgeon's satisfaction. The result was considered good or satisfactory when it showed minimal remaining gland, good symmetry, no retraction, necrosis, hypertrophic scar, or displacement of the nipple-areola complex. All patients answered a questionnaire to evaluate their satisfaction and perception of the procedure.

\section{RESULTS}

Seven patients with Simon grade 1 and 2 bilateral GM underwent ultrasound-guided VAE. One of them had undergone previous traditional open surgical treatment of GM with unsatisfactory results, and all patients expressed their wish to have an excision with less morbidity, small scars, and good esthetic outcome. The mean age was 27.5 years (ranging from 19 to 34 years). The average procedure time was 28 minutes (ranging from 23 to 54 minutes). The main complaint and indication for the procedure was the esthetic appearance of GM, followed by physical deformity. One patient had an areola fissure caused by the vacuum suction during the procedure, which was promptly sutured and did not affect the final esthetic result. At followup, all patients and breast surgeons reported excellent or good satisfaction (Figures 4 and 5), and at the six-month review, no patient presented recurrence or asked for another intervention or open surgery. No patient had postoperative seroma, bleeding, or hemorrhage or needed to be taken to the operating room at any time, during or after the surgical procedure and follow-up. All procedures were performed in an outpatient setting, with local anesthesia and no sedation. Histological evaluation revealed benign GM in all patients. No case of displacement, necrosis, or 

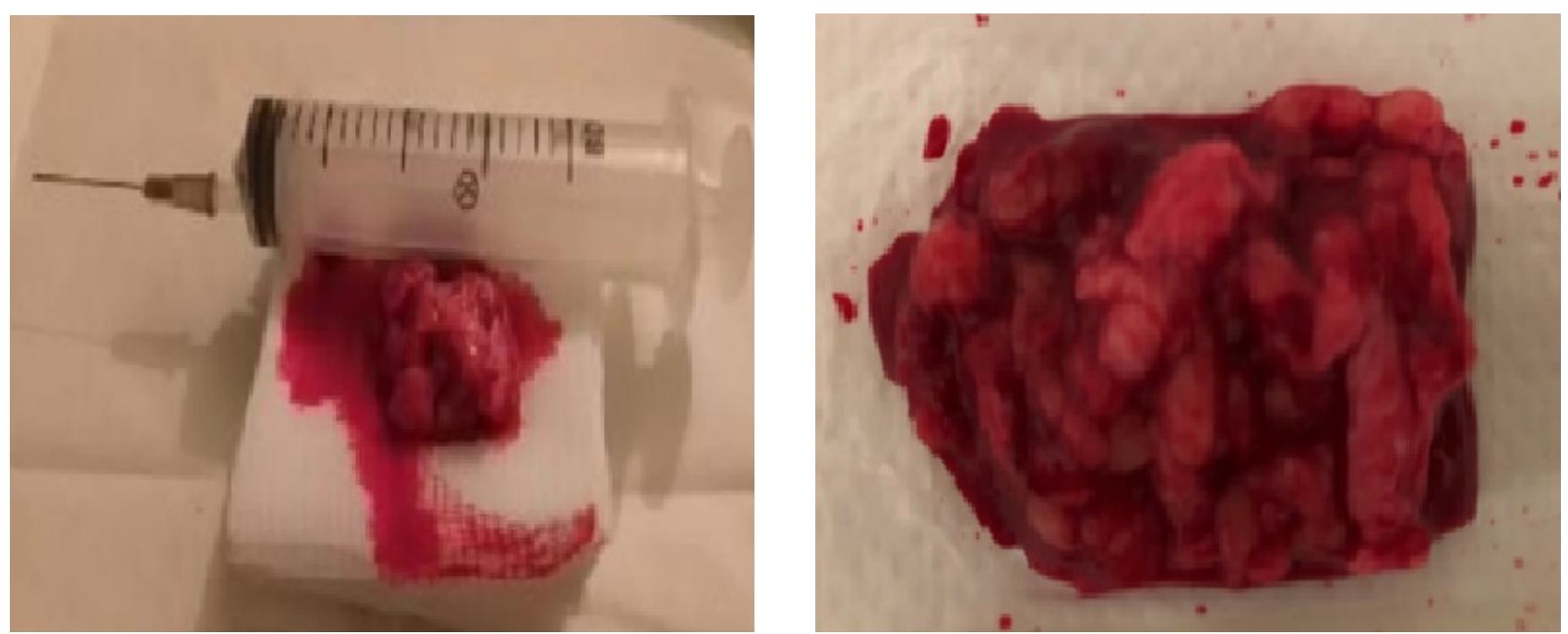

Figure 2. Ultrasound-guided vacuum-assisted excision of gynecomastia: surgical specimen.
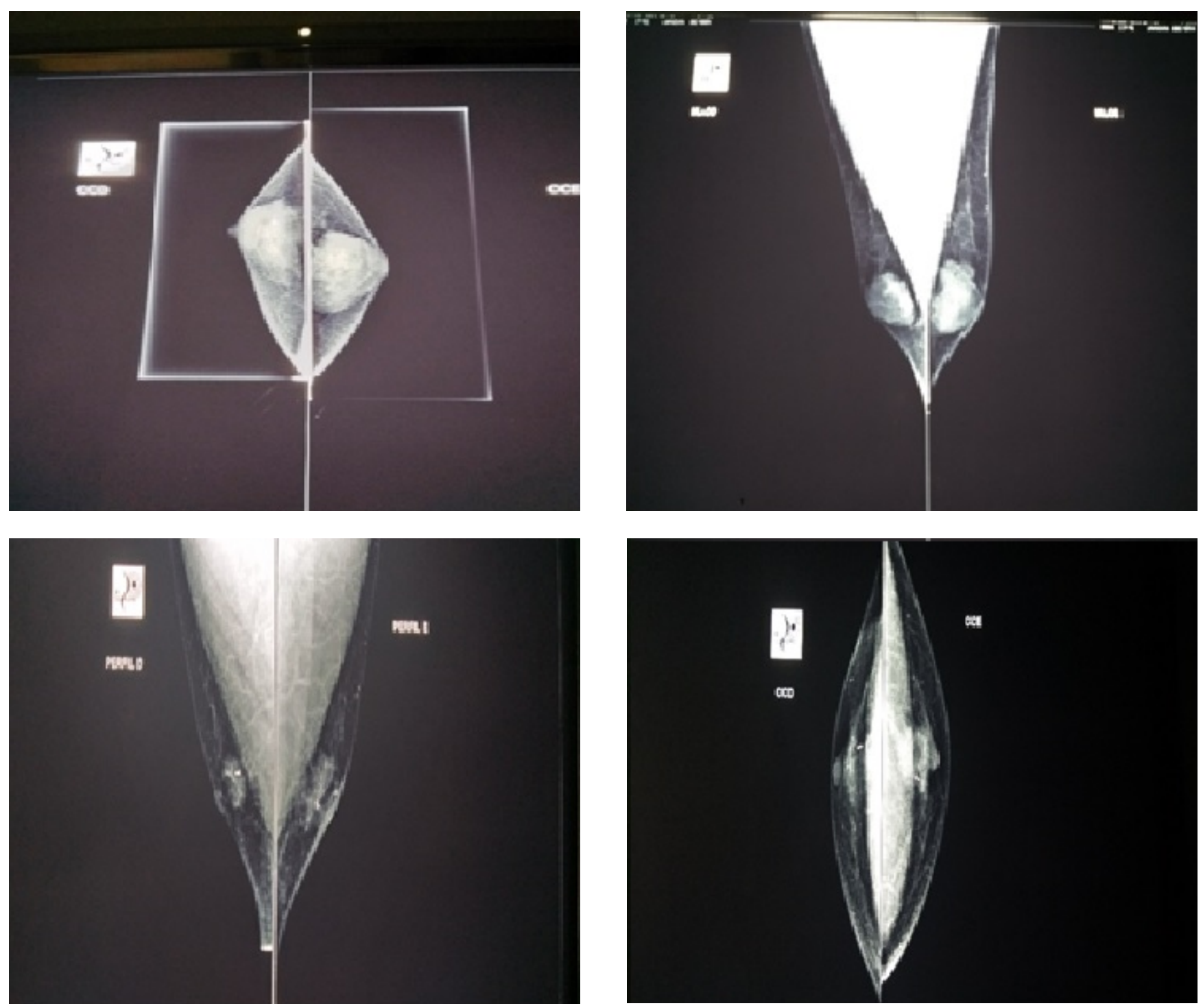

Figure 3. Mammograms before and six months after ultrasound-guided vacuum-assisted excision of gynecomastia. 
retraction of the nipple-areola complex was detected. None of the individuals investigated presented postoperative bleeding, infection, skin necrosis, or asymmetry. No patient reported decrease or change in nipple sensation or erection. All patients had bruises and hematomas that spontaneously resolved within 30 days of VAE, with excellent or good cosmetic results and no skin sequelae. The individuals investigated were able to return to their life activities in 2 days and to physical work in 14 days. Physical activities were allowed two weeks after the procedure. All results were considered good or excellent by patients and surgeons (Table $2^{13}$ and Figure 3).

\section{DISCUSSION}

The main goal of treating GM is to remove the excess of breast tissue, achieving the best symmetry with minimal scarring and good or excellent esthetic results. Different from subcutaneous mastectomy for cancer treatment, the purpose of GM surgery is not to excise all breast tissue in an oncologic fashion. GM surgery aims to remove enough breast tissue to obtain a good cosmetic result and avoid clinical recurrence. The open surgical approach is still the standard procedure for persistent GM after one or two years, especially when associated with psychological distress, unsatisfactory body image, and avoidance of activities in which the chest is exposed (sports and swimming) ${ }^{4}$. For years, subcutaneous mastectomy through a semicircular inferior areolar incision, associated or not with liposuction, has been the gold-standard surgical

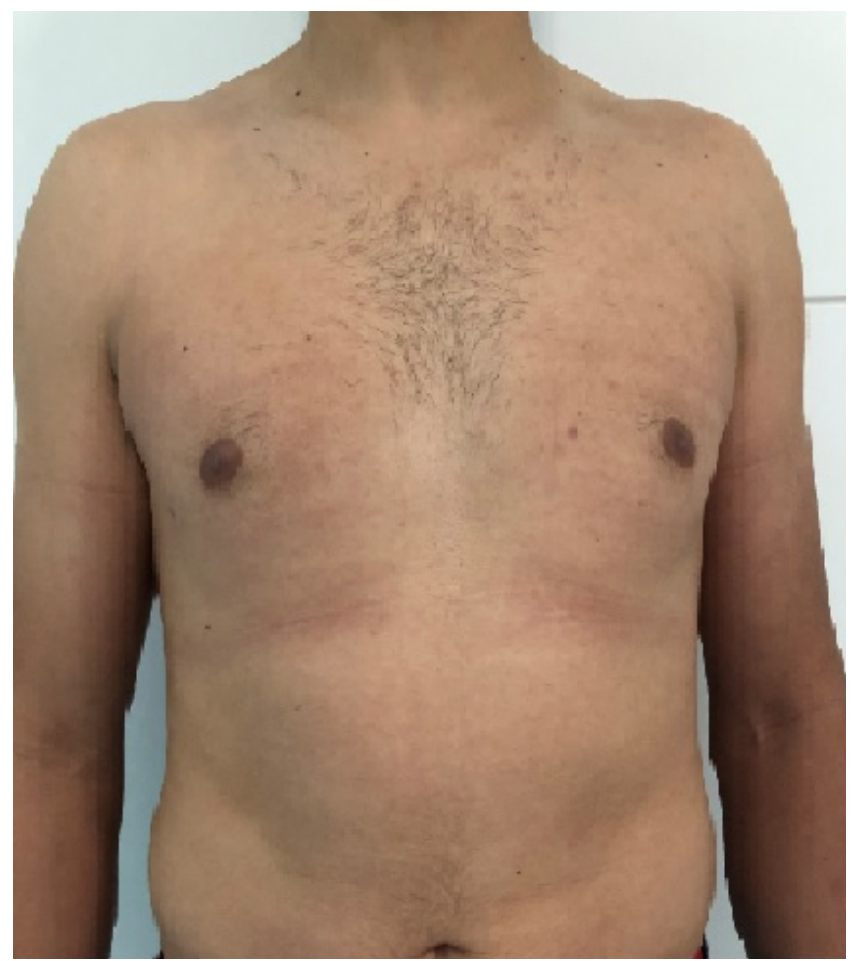

Figure 4. 34-year-old man with Simon grade 2 gynecomastia. procedure for this condition. The results are usually satisfactory, but postoperative complications are common, including areola deformity or retraction; "saucer-shaped defect" (from over-resection of breast tissue); seroma; poor scarring, such as retraction, hypertrophic scar, or keloid formation; wound dehiscence; and nipple retraction, necrosis, or altered sensation. The side effects of standard surgery have been a longstanding concern. In 1987, Courtiss et al. published an article reporting that 101 out of 159 patients presented high complication rates after traditional excision for the treatment of GM, including under-resection (21.9\%), "saucer-shaped defect" (18.7\%), poor scarring (18.7\%), hematoma (16.1\%), and seroma $(9.4 \%)^{6}$. In order to decrease morbidity and improve esthetic results, the GM treatment should improve with new surgical techniques and minimally invasive procedures.

More recently, some groups have described an endoscopeassisted subcutaneous mastectomy ${ }^{5}$, with a smaller incision. However, this technique did not eliminate the potential complication of having a scar on a visible part of the chest or axillae, and the risk of nipple-areola complex complications remains 8 .

In 2010, the Royal College of Surgeons of England published the first article about a vacuum-assisted biopsy device associated with liposuction to provide a minimally invasive approach for GM, with excellent results ${ }^{8}$. The group suggested that ultrasound guidance could be positive in those cases. One year later, the Chinese experience with a vacuum-assisted biopsy device was also published ${ }^{9}$. Recently, the indications

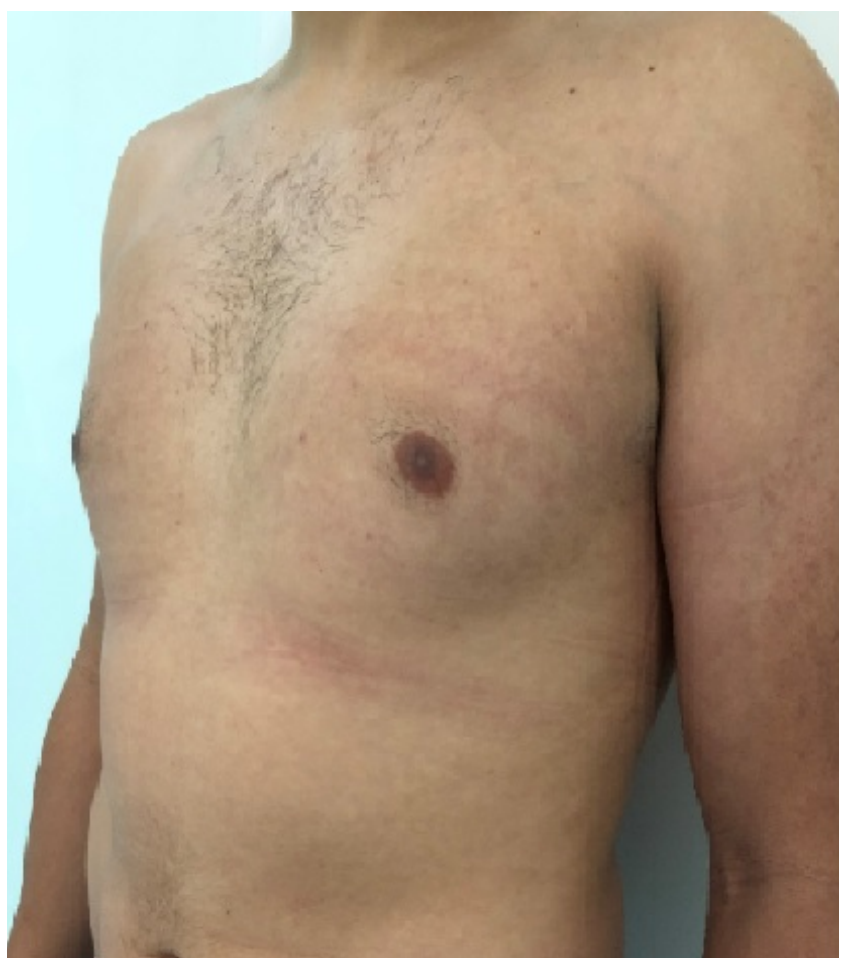


for VAE have expanded to more severe Simon grades of GM, with the procedure performed in the operating room under general anesthesia ${ }^{10}$.

A recent prospective series compared VAE of GM with open traditional surgery. The VAE group had significantly smaller scar sizes $(0.40 \pm 0.08 \mathrm{~cm}$ vs. $5.34 \pm 0.38 \mathrm{~cm}, \mathrm{p}<0.01)$, shorter healing time (3.67 \pm 0.71 days vs. $7.90 \pm 0.92$ days, $p<0.01)$ and hospitalization ( $2.60 \pm 0.62$ vs. $7.17 \pm 0.83$ days, $p<0.01$ ), as well as higher postoperative satisfaction $(4.70 \pm 0.60$ scores vs. $3.20 \pm 0.55$ scores, $\mathrm{p}<0.01$ ). The incidence rate of bruises was significantly higher in the VAE group compared to the open surgical group ( $47 \%$ vs. $17 \%, p=0.013$ and $54 \%$ vs. $20 \%, p=0.007)$, respectively ${ }^{11}$.
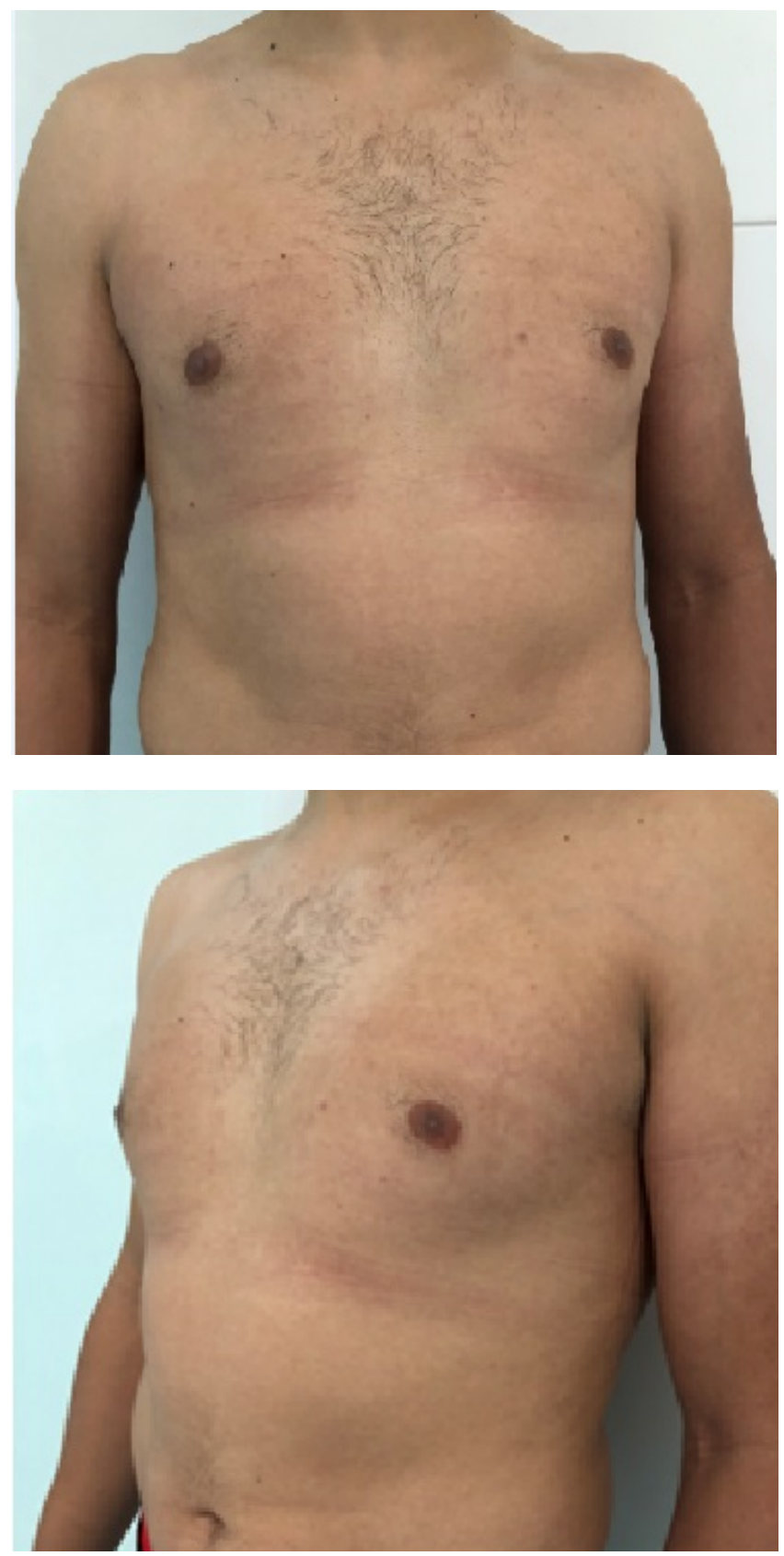

The benefits of VAE are similar to those of minimally invasive procedures in general - reduced morbidity, better esthetic results, fewer recovery days, and no hospitalization time or $\operatorname{cost}^{8}$. The results from this series corroborate the findings of other series and studies. Depending on the GM grade, the VAE can be performed with local anesthesia, with or without sedation. With the evolution of vacuum-assisted devices, better vacuum aspiration, and multiple fragments collected in an automated circular approach with one-step needle insertion, it is possible to remove a considerable amount of breast tissue in a few minutes, reducing the odds of infection or complication. A study reported a median time of 50
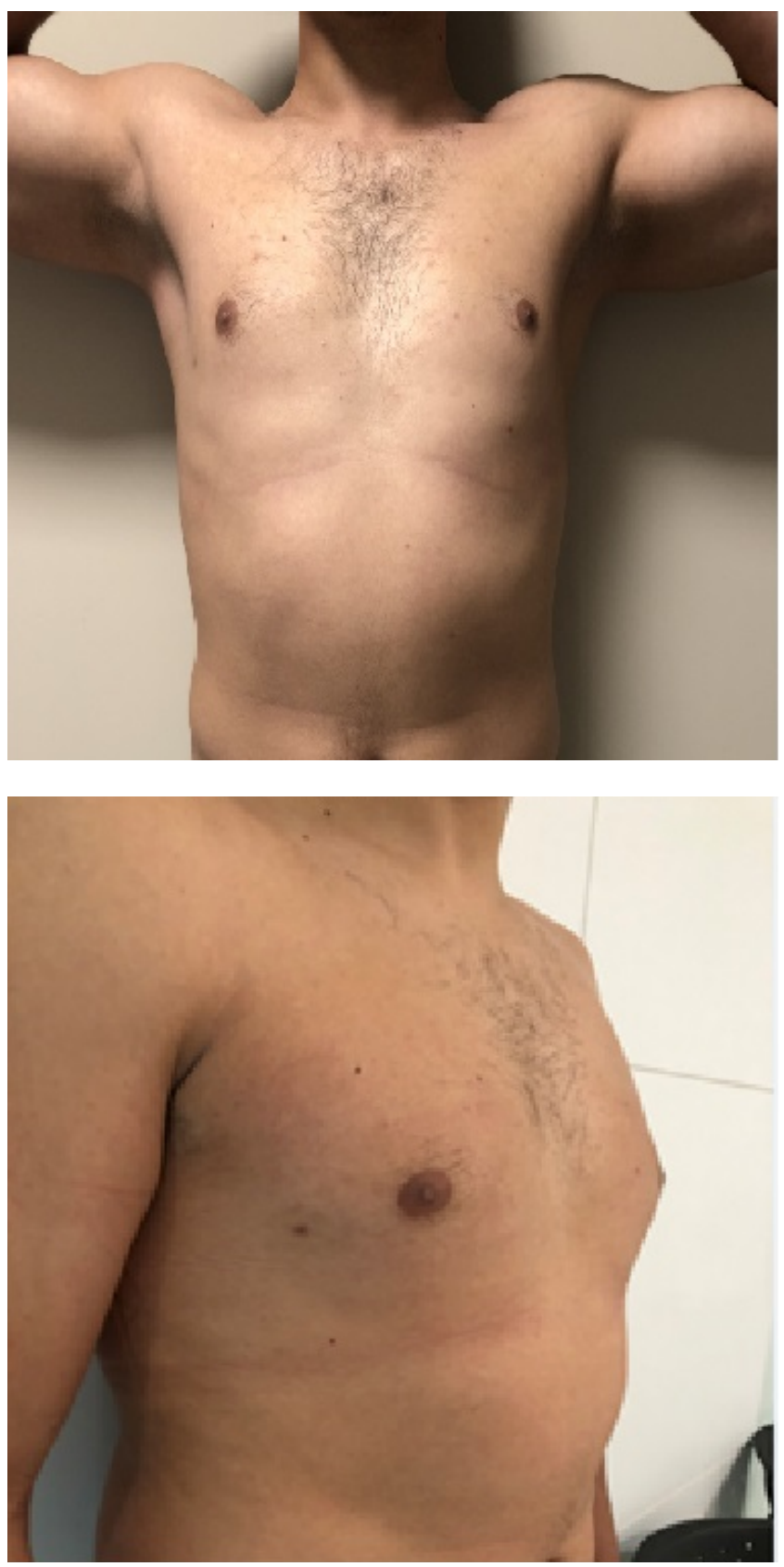

Figure 5. Same patient six months after ultrasound-guided vacuum-assisted excision of gynecomastia. 
Table 2. Satisfaction evaluation: adaptation of the consultation satisfaction questionnaire.

\begin{tabular}{|c|c|c|c|c|}
\hline $\mathrm{n}=7$ & Esthetic discomfort & Physical deformity & Medical indication & \\
\hline Patient complaint & 5 & 2 & 0 & \\
\hline $\mathrm{n}=7$ & Excellent & Good & Regular & Bad \\
\hline Final esthetic result (6 months) - patient & 5 & 2 & 0 & 0 \\
\hline $\begin{array}{l}\text { Final esthetic result ( } 6 \text { months) - } \\
\text { surgeon }\end{array}$ & 4 & 3 & 0 & 0 \\
\hline$n=7$ & yes & no & & \\
\hline $\begin{array}{l}\text { Would the patient repeat or recommend } \\
\text { the procedure for someone? }\end{array}$ & 7 & 0 & & \\
\hline Was the procedure well tolerated? & 7 & 0 & & \\
\hline \multicolumn{5}{|l|}{ Complications $n=7$} \\
\hline Seroma & 0 & & & \\
\hline Bruises & 7 & & & \\
\hline Anesthesia scar & 0 & & & \\
\hline Bleeding & 0 & & & \\
\hline Areola fissure & 1 & & & \\
\hline $\begin{array}{l}\text { Displacement, necrosis, or retraction of } \\
\text { the nipple-areola complex. }\end{array}$ & 0 & & & \\
\hline $\begin{array}{l}\text { Decrease or change in nipple sensation } \\
\text { or erection }\end{array}$ & 0 & & & \\
\hline
\end{tabular}

Source: Mazzarone ${ }^{13}$

minutes using an $8 \mathrm{G}$ needle with a semi-automated device ${ }^{8}$, while in this series, the median time was 25 minutes using a $10 \mathrm{G}$ needle with a whole circumference automated device. The patients' procedure tolerance was high, even with just local anesthesia Automated devices allow faster, safe, and outpatient procedures that preclude hospitalization and have the potential of saving costs.

Doubts related to long-time recurrence remain and require more studies for clarification. Longer follow-up will be necessary to evaluate this issue better. Nevertheless, the amount of breast tissue excised described by the literature and this series is not different from the traditional open surgical specimen. Mammographic images gradually change over time. After six months, it is possible to estimate the amount of tissue resected, but, like in benign surgeries, the degree of architectural distortion is high, especially due to large hematomas and bruises, which fade with time. This finding indicates that the best moment for a mammographic evaluation of the amount of breast resected should probably be after one year of the procedure.

\section{CONCLUSION}

Minimally invasive ultrasound-guided VAE is an excellent alternative for the treatment of GM. It is better indicated for Simon grade 1 and 2 GM, with good and excellent esthetic results and low rates of nipple and areolar complications. It allows an outpatient procedure with low morbidity (local anesthesia) and fast recovery. Hematomas and bruises are always present due to the nature of the approach. Breast surgeons can obtain satisfactory cosmetic results with little morbidity and postoperative complications, such as nipple retraction or necrosis. Ultrasound-guided VAE has become a valuable approach for the surgical management of Simon grade 1 and 2 GM, with or without liposuction according to necessity. Trials comparing VAE of GM with open surgery should also evaluate clinically relevant recurrence throughout the years to establish the safety of these surgical approaches over time.

\section{AUTHORS' CONTRIBUTION}

C.V.: Investigation, Methodology, Project Administration, Writing - Review and Editing.

H.L.: Investigation, Methodology, Project Administration, Supervision, Validation, Writing - Review and Editing. T.O.: Writing - Review and Editing, Formal Analysis.

P.B.: Methodology, Writing - Review and Editing.

S.F.: Data Curation, Validation, Writing - Review and Editing. O.J.: Investigation, Visualization, Writing - Original Draft, Validation. 


\section{REFERENCES}

1. Kanakis GA, Nordkap L, Bang AK, Calogero AE, Bártfai G, Corona $\mathrm{G}$, et al. EAA clinical practice guidelines-gynecomastia evaluation and management. Andrology. 2019;7(6):778-93. https://doi.org/10.1111/andr.12636

2. Narula HS, Carlson HE. Gynaecomastia: pathophysiology, diagnosis and treatment. Nat Rev Endrocrinol. 2014;10(11):68498. https://doi.org/10.1038/nrendo.2014.139

3. Vojvodic M,Xu FZ, Cai R, Roy M, FieldingJC.Anabolic-androgenic Steroid Use Among Gynecomastia Patients: Prevalence and RelevancetoSurgicalManagement.AnnPlastSurg.2019;83(3):25863. https://doi.org/10.1097/SAP.0000000000001850

4. Simon BE, Hoffman S, Kahn S. Classification and surgical correction of gynecomastia. Plast Reconstr Surg. 1973;51(1):4852. https://doi.org/10.1097/00006534-197301000-00009

5. Varlet F, Raia-Barjat T, Bustangi N, Vermersch S, Scalabre A. Treatment of Gynecomastia by Endoscopic Subcutaneous Mastectomy in Adolescents. J Laparoendosc Adv Surg Tech A. 2019;29(8):1073-6. https://doi.org/10.1089/lap.2019.0256

6. Courtiss EH. Gynecomastia: analysis of 159 patients and current recommendations for treatment. PlastReconstrSurg. 1987;79(5):74053. https://doi.org/10.1097/00006534-198705000-00010

7. Colombo-Benkmann M,Buse B, SternJ,Herfarth C.Indications for and results of surgical therapy for male gynecomastia. Am J Surg. 1999;178(1):60-3. https://doi.org/10.1016/s0002-9610(99)00108-7
8. Qutob O, Elahi B, Garimella V, Ihsan N, Drew PJ. Minimally invasive excision of gynaecomastia-a novel and effective surgical technique. Ann R Coll Surg Engl. 2010;92(3):198-200. https://doi.org/10.1308/003588410x12628812458815

9. He Q, Zheng L, Zhuang D, Fan Z, Xi C, Zhou P. Surgical treatment of gynecomastia by vacuum-assisted biopsy device. J Laparoendosc Adv Surg Tech A. 2011;21(5):431-4. https://doi. org/10.1089/lap.2011.0019

10. Yao Y, Yang Y, Liu J, Wang Y, Zhao Y. Vacuum-assisted minimally invasive surgery. An innovative method for the operative treatment of gynecomastia. Surgery. 2019;166(5):9349. https://doi.org/10.1016/j.surg.2019.04.032

11. WangY,WangJ,LiuL,LiangW,QinY,ZhengZ, etal.Comparison of curative effects between mammotome-assisted minimally invasive resection (MAMIR) and traditional open surgery for gynecomastia in Chinese patients: A prospective clinical study. Breast J. 2019;25(6):1084-9. https://doi.org/10.1111/ tbj. 13424

12. Iwuagwu O,DrewP.Vacuum-assisted biopsy device-diagnostic and therapeutic applications in breast surgery. Breast. 2004;13(6):483-7. https://doi.org/10.1016/j.breast.2004.06.004

13. Mazzarone F. Avaliação da satisfação do resultado de cirurgia plástica [dissertation]. Rio de Janeiro: Fundação Cesgranrio; 2013. 